EPJ Web of Conferences 21, 02002 (2012)

DOI: $10.1051 /$ epjconf/20122102002

(C) Owned by the authors, published by EDP Sciences, 2012

\title{
Clustering effects in ${ }^{48} \mathrm{Cr}$ composite nuclei produced via ${ }^{24} \mathrm{Mg}$ $+{ }^{24} \mathrm{Mg}$ reaction
}

\author{
A. Di Nitto ${ }^{1, a}$, A. Brondi ${ }^{2}$, G. La Rana ${ }^{2}$, R. Moro ${ }^{2}$, P. Nadtochy ${ }^{1}$, E. Vardaci ${ }^{2}$, A. Vanzanella ${ }^{1}$, M. \\ Cinausero $^{3}$, G. Prete ${ }^{3}$, and N. Gelli ${ }^{4}$ \\ 1 Istituto Nazionale di Fisica Nucleare,Via Cinthia, 80126 Napoli, Italy \\ 2 Istituto Nazionale di Fisica Nucleare and Dipartimento di Scienze Fisiche, Universitá di Napoli \\ "Federico II", Via Cinthia, 80126 Napoli, Italy \\ 3 Laboratori Nazionali di Legnaro dell'Istituto Nazionale di Fisica Nucleare, Legnaro (Padova), Italy \\ 4 Istituto Nazionale di Fisica Nucleare, Firenze, Italy
}

\begin{abstract}
In the framework of studying clustering effects in $\mathrm{N}=\mathrm{Z}$ light nuclei, an experiment was carried out to get information on the properties of the ${ }^{48} \mathrm{Cr}$ composite nuclei produced via the ${ }^{24} \mathrm{Mg}+{ }^{24} \mathrm{Mg}$ reaction. In particular, the study regards the ${ }^{48} \mathrm{Cr}$ at 60 $\mathrm{MeV}$ of excitation energy where a resonance with a narrow width $(170 \mathrm{KeV})$ has been found by measuring the elastic and anelastic channels. To determine the deformation of this state, evaporative Light Charged Particles (LCP) are measured and compared to the Statistical Model (SM) predictions, which are very sensitive to nuclear deformation. The experiment was performed at LNL using the $8 \pi \mathrm{LP}$ apparatus to select LCPs and a Parallel Plate Avalanche Counter (PPAC) system to detect the Evaporation Residues (ER). Preliminary results on the measurements of $E R-L C P$ and $L C P-L C P$ angular correlations are presented which indicate the presence of a very large deformation.The analysis will proceed with the extraction of the LCP energy spectra and with the angular correlations for high multiplicity channels to determine the deformation.
\end{abstract}

\section{Introduction}

The phenomena of clustering in $\mathrm{N}=\mathrm{Z}$ light nuclei have recently raised much interest [1-3]. These phenomena have been enlightened by the observation of relatively narrow resonances $(\Gamma \approx 100-200$ $\mathrm{keV}$ ) in the elastic and inelastic channels for $\alpha$-like nuclei [4]. Formation of highly deformed systems, with lifetime comparable to that of compound nucleihas been evidenced.

Although the nature and the reaction mechanism involved in these reactions are not yet completely understood, different interpretations have been proposed. They rely on the formation of states with very large deformations in the dinuclear system, acting as doorway states to fusion, with a strong memory of the entrance channel. In this respect, entrance channel dynamics may affect the evaporative decay if the dinuclear complex forms a highly deformed configuration, as observed in reaction involving $\alpha$-nuclei, with lifetime comparable to the evaporation time scale. Therefore, evaporative light charged particles can be a good probe to estimate the deformation of these states.

As a matter of fact, using as probe the light charged particles, large nuclear deformations were found for the nuclei: ${ }^{58} \mathrm{Ni}[5],{ }^{32} \mathrm{~S} \mathrm{[6]} \mathrm{and}{ }^{40} \mathrm{Ca}[7,8]$, produced in reactions between $\alpha$-nuclei. Concerning ${ }^{58} \mathrm{Ni}$, the analysis of light particle energy spectra and angular correlations, in the framework of the statistical model, indicates the onset of large nuclear deformations at high spin, significantly larger than those predicted by the Rotating Liquid Drop Model (RLDM) [9]. It must be pointed out that in this case, the critical angular momentum for fusion was $34 \hbar$, a value lower than the resonance angular

\footnotetext{
a e-mail: dinitto@na.infn.it
}

This is an Open Access article distributed under the terms of the Creative Commons Attribution-Noncommercial License 3.0, which permits unrestricted use, distribution, and reproduction in any noncommercial medium, provided the original work is properly cited. 


\section{EPJ Web of Conferences}

momentum by $4 \hbar$. Therefore, an open question is whether or not such kind of resonances could act as doorway states for fusion at angular momenta higher than the critical angular momentum. However, as indicated by the authors [5], the extent to which the resonance is responsible for the observed nuclear deformation is still an open question.

A deformation significantly larger than that predicted by the RLDM was also observed for the nucleus ${ }^{32} \mathrm{~S}$. In particular, this deformation is significantly higher than those observed for the non $\alpha$ like systems ${ }^{19} \mathrm{~F}+{ }^{12} \mathrm{C}$ and ${ }^{20} \mathrm{Ne}+{ }^{27} \mathrm{Al}$, at comparable excitation energies, even though in the ${ }^{20} \mathrm{Ne}+$

${ }^{27} \mathrm{Al}$ reaction the angular momentum involved is much higher.

Finally, deformations far in excess of those predicted by the RLDM [9] have also been found for the nucleus ${ }^{40} \mathrm{Ca}$.

Narrow resonance phenomena in the elastic and inelastic channels have been observed for the system ${ }^{24} \mathrm{Mg}+{ }^{24} \mathrm{Mg}$ at high $\operatorname{spin}(\mathrm{J}=34-36 \hbar)$ and at high excitation energy $\left(\mathrm{E}_{\mathrm{x}}=60 \mathrm{MeV}\right)$. In particular, a narrow resonance with $\mathrm{J}=36 \hbar$ at $\mathrm{E}_{\mathrm{x}}=60 \mathrm{MeV}[10,11]$ was observed. The resonance spin is higher than the grazing angular momentum in the entrance channel $\left(\mathrm{L}_{\text {graz }}=32 \hbar\right)$ by $4 \hbar$, raising many questions on this subject. Furthermore, the width of $170 \mathrm{keV}$ implies a lifetime of $4 \times 10^{-21} \mathrm{~s}$, ten times longer than a typical nuclear lifetime. A study has been recently carried out [12] on the $\mathrm{J}=36 \hbar^{24} \mathrm{Mg}$ $+{ }^{24} \mathrm{Mg}$ resonance, in order to investigate its decay into the inelastic and fusion-evaporation channels. The measurements of ON and OFF resonance decay yields allowed to enlighten a strong resonant effect in the inelastic channels and, to a minor extent, in certain fusion-evaporation channels. The properties of the studied resonance are in agreement with the molecular model predictions [13] and are very close to those for a highly prolate deformed ${ }^{48} \mathrm{Cr}$ after a Jacobi shape transition, predicted by the Lublin - Strasbourg - Drop (LSD) model [14] between J=28 $\hbar$ and $32 \hbar$, at Ex $=60 \mathrm{MeV}$.

In this framework, we have undertaken the study of the nuclear deformation of the compound nucleus ${ }^{48} \mathrm{Cr}$, produced at $\mathrm{E}_{\mathrm{x}}=60 \mathrm{MeV}$ by $\mathrm{E}_{\mathrm{lab}}=91.7 \mathrm{MeV}{ }^{24} \mathrm{Mg}+{ }^{24} \mathrm{Mg}$ reactios, using as a probe the light charged particles. The fusion cross section at this energy is $\sigma_{\text {fus }}=1060 \mathrm{mb}[15,16]$ implying a fusion angular momentum $\mathrm{L}_{\text {fus }} \approx 30 \hbar$. At this excitation energy, possible clustering effects on the decay of ${ }^{48} \mathrm{Cr}$ composite systems are expected, with the occurrence of large deformations, as already observed for other systems produced by $\alpha$-like nuclei. Comparisons of the data with the predictions of the statistical model is expected to be particularly elucidating for this investigation.

\section{Experimental setup}

The experiment was performed at the Tandem accelerator of Laboratori Nazionali di Legnaro. A pulsed beam of ${ }^{24} \mathrm{Mg}$ of intensity of about 5-10 enA was used to bombard a ${ }^{24} \mathrm{Mg}$ target $60 \mu \mathrm{g} / \mathrm{cm}^{2}$ thick on $15 \mu \mathrm{g} / \mathrm{cm}^{2} \mathrm{C}$ backing. A beam burst with period of $800 \mathrm{~ns}$ and duration of about $3 \mathrm{~ns}$ was used.

The chosen beam energy of $91.4 \mathrm{MeV}$ corresponds to the resonance $\mathrm{J}^{\pi}=36 \hbar^{+}$at $\mathrm{E}_{\mathrm{x}}=60 \mathrm{MeV}$ in the ${ }^{48} \mathrm{Cr}$ compound nucleus $(\mathrm{CN})$. The light charged particles emitted from the $\mathrm{CN}$ de-excitation were measured with the $8 \pi \mathrm{LP}$ apparatus [17].

The detector system, shown schematically in fig. 1 (left side), is constituted by about 250 telescopes made by a first stage $\Delta \mathrm{E}$ of Si detectors followed by a second stage of CsI detectors, geometrically arranged in two sections: the BALL and the WALL.

Particle identification was carried out by using the $\Delta \mathrm{E}-\mathrm{E}$ technique for the particles that have energy enough to pass through the $\Delta \mathrm{E}$ stage. To identify the particles that stop in the Si detectors different techniques were used, i.e. Pulse Shape Discrimination for the BALL detectors and time of flight (ToF) for the WALL ones. For the reaction under study we were able to identify the charged particles with energy thresholds of $0.5 \mathrm{MeV}$ for protons and $1 \mathrm{MeV}$ for alpha particles.

Data presented at the conference refer to the BALL section of the apparatus. The BALL has a diameter of $30 \mathrm{~cm}$ and consists of 7 rings of detectors placed coaxially around the beam direction. Each ring, labeled from A to $\mathrm{G}$ going from backward to forward angles, contains 18 telescopes pointing towards the center of the target, and covers an angular opening of about $17^{\circ}$. The BALL has a total of 126 telescopes and covers the polar angle from $34^{\circ}$ to $165^{\circ}$. Considering this geometry, the detectors in a ring have the same average polar angle with respect to the beam direction axis, and all together 
CNR*11
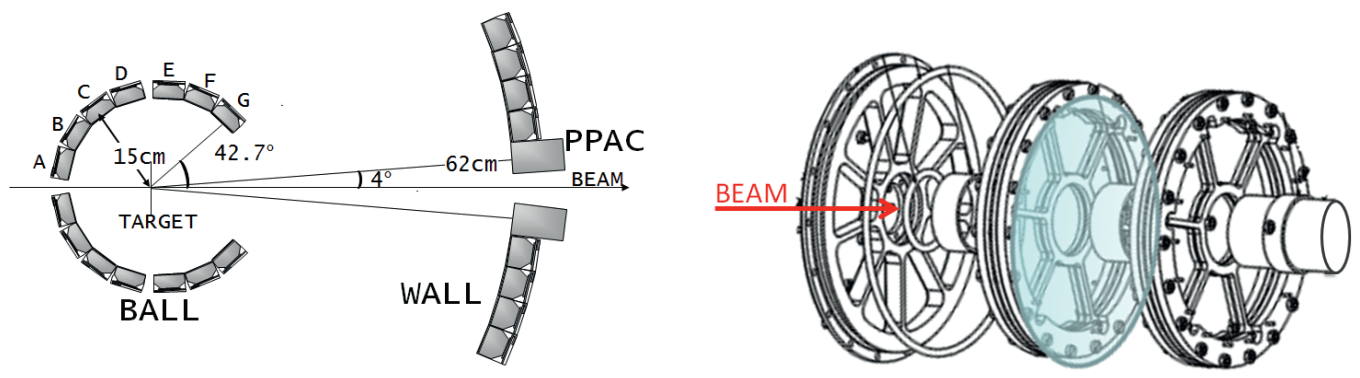

Fig. 1. Schematic layout of the experimental apparatus. The letters from A to $G$ label the 7 rings of the BALL section (left side). Scheme of the PPAC system (right side).

cover the azimuthal angle from $0^{\circ}$ to $360^{\circ}$. As a whole the BALL covers a solid angle of about $80 \%$ of $4 \pi$.

In the present experiment, evaporation residues were detected by a circular PPAC system divided in 6 independent sectors, fig. 1 (right side). It is placed in the forward direction at $62 \mathrm{~cm}$ from the target.

In order to define a narrow angular selection of ERs, in front of each sector a mask with a hole $1 \mathrm{~cm}$ in diameter was mounted. Each hole subtended a solid angle of $0.8 \mathrm{msr}$ and was centered at $4^{\circ}$ with respect to the beam direction. The PPAC system consists of two coaxial PPACs (front and rear), with a propilene foil $35 \mu \mathrm{m}$ thick mounted in between. This thickness was sufficient to stop the ERs between the two PPACs, and let the other ions, like LCP and elastic scattered beam particles, reach the rear PPAC. The ERs were therefore selected using as a veto the rear PPAC signal.

The LCPs and the ERs events were collected. We used different trigger conditions to select the observables more sensitive to the nuclear deformation according to the results of the SM simulations.

Data were collected requiring the OR mode between the following conditions: a) events detected by any PPAC module or by any LCP detectors to select in single mode ER and LCP events, respectively b) coincidences between any PPAC sector and any particle detector to select events corresponding to the light particles emitted in the ER channel, and c) two-fold, three-fold and four-fold events in the particle detectors to select the channels with high multiplicity. This triggering scheme was very effective for the evaluation of the multiplicities because it provided, in the same run, for the measurement of the singles and coincidence yields.

\section{Statistical model simulations}

In this work the SM calculations were performed with the code LILITA_N97 [18]. The computer code LILITA models the multistep evaporative decay of a compound nucleus by using the Hauser-Feshbach formulation of the SM in conjunction with the Monte Carlo method. The emission of neutrons, protons and alpha particles is taken into account while fgission is not considered. The program produces laboratory frame energy spectra and angular distributions for both ER and light particles, once the constraints imposed by the experimental apparatus are included.

The code LILITA_N97 is an extensively modified version of the original LILITA code. New options were included for the transmission coefficients and the level density. Transmission coefficients can be chosen between the optical model (OM) [20-22] and the fusion systematics (FS) [23]. Furthermore, modified versions of the Fermi gas level density with different prescriptions of the level density parameter were implemented along with the following prescriptions for the yrast line: 1) with parameters from the Rotating Liquid Drop Model (RLDM) [9], 2) the Gilbert-Cameron prescription [24], 3) with parameters provided by the user.

In this study we simulated the LCP decay from a normal and highly deformed ${ }^{48} \mathrm{Cr}$ composite system using two sets of deformability parameters $\delta_{1}$ and $\delta_{2}$ to modulate the deformation. These parameters are usually adopted in the statistical model to calculate the moment of inertia $\mathfrak{I}$ of the 


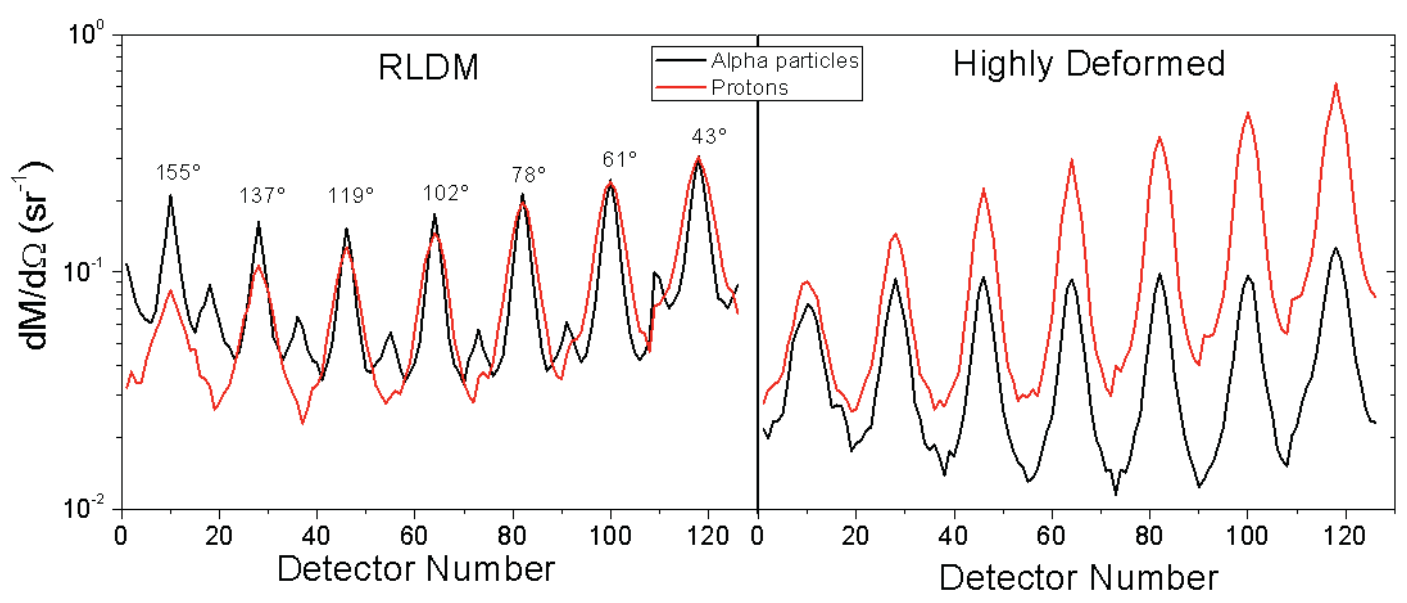

Fig. 2. Simulated angular multiplicity distributions of protons and alpha particles in coincidence with ERs versus the identification number of the BALL detectors for RLDM (left side) and Highly Deformed (right side) calculations.

emitting nucleus as a function of the angular momentum $\mathrm{J}$, through the expression:

$$
\mathfrak{J}=\mathfrak{J}_{0} \cdot\left(1+\delta_{1} \mathbf{J}^{2}+\delta_{2} \mathbf{J}^{4}\right)
$$

The first calculation adopted a set of deformability parameters obtained according to the RLDM (corresponding to a deformation with an axis ratio $\mathrm{b} / \mathrm{a}=1.51$ for $\mathrm{J}=36 \hbar$ ) with spherical FS transmission coefficients. The second calculation, indicated as Highly Deformed, was obtained with the following prescription: For the angular momenta in the window $\mathrm{J}=28 \hbar-36 \hbar$ (around the angular momentum of the resonance) very large deformations were used $(\mathrm{b} / \mathrm{a}=3.0)$, with deformed emission barriers taken from systematics [25]. For lower angular momenta the RLDM deformation and spherical FS transmission coefficients was used.

The geometry of $8 \pi \mathrm{LP}$ apparatus coupled to the PPAC system was included in the simulations.

In order to look for the sensitivity of the observables to the deformation, we have performed the simulations of $E R-L C P$ and $L C P-L C P$ coincidences.

In fig. 2 the differential multiplicities for protons and alpha particles in coincidence with one PPAC sector are shown as a function of the BALL detector number starting from the most backward angle. In the figure the polar angle of each ring of the BALL is indicated. On the left and right side of the figure the results corresponding to RDLM and to Highly Deformed deformations are shown, respectively.As mentioned before, for a fixed polar angle, the detectors span in azimuthal angles from 0 to $360^{\circ}$ giving rise to peaks in the angular correlation. This behaviour is due to a combined effect ofthe reaction kinematics and of the angular momentum of the composite system. In particular, the maxima correspond to the events where ERs and LCPs are in-plane on the opposite side with respect to the beam direction, while the minima occur when ERs and LCPs are in-plane on the same side with respect to the beam direction.

As it can be inferred from the figure, $E R-L C P$ angular correlations are very sensitive to the nuclear deformation. The effects of a large nuclear deformation appear as:

- a significant change in the proton to alpha-particle multiplicity ratio;

- a significant increase of the maximum to minimum ratio for the detectors of the same ring;

- the disappearing of the small peaks present in the RLDM alpha-particle correlation.

\section{Data analysis: preliminary results}

We present a preliminary analysis of the measured $E R-L C P$ and $L C P-L C P$ coincidences comparing the experimental data to the LILITA_N97 predictions. Our goal was to find an indication of 
$\mathrm{CNR} * 11$

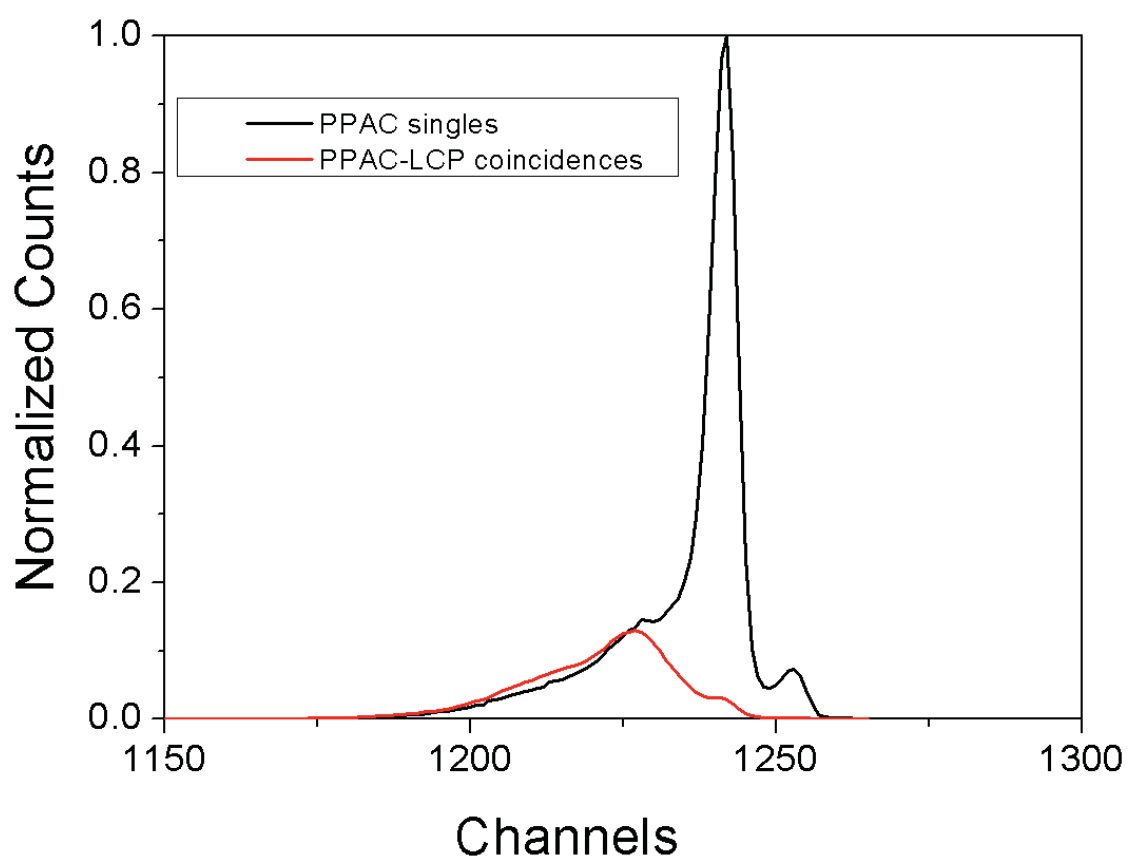

Fig. 3. Time spectrum of fragments detected in one PPAC sector.

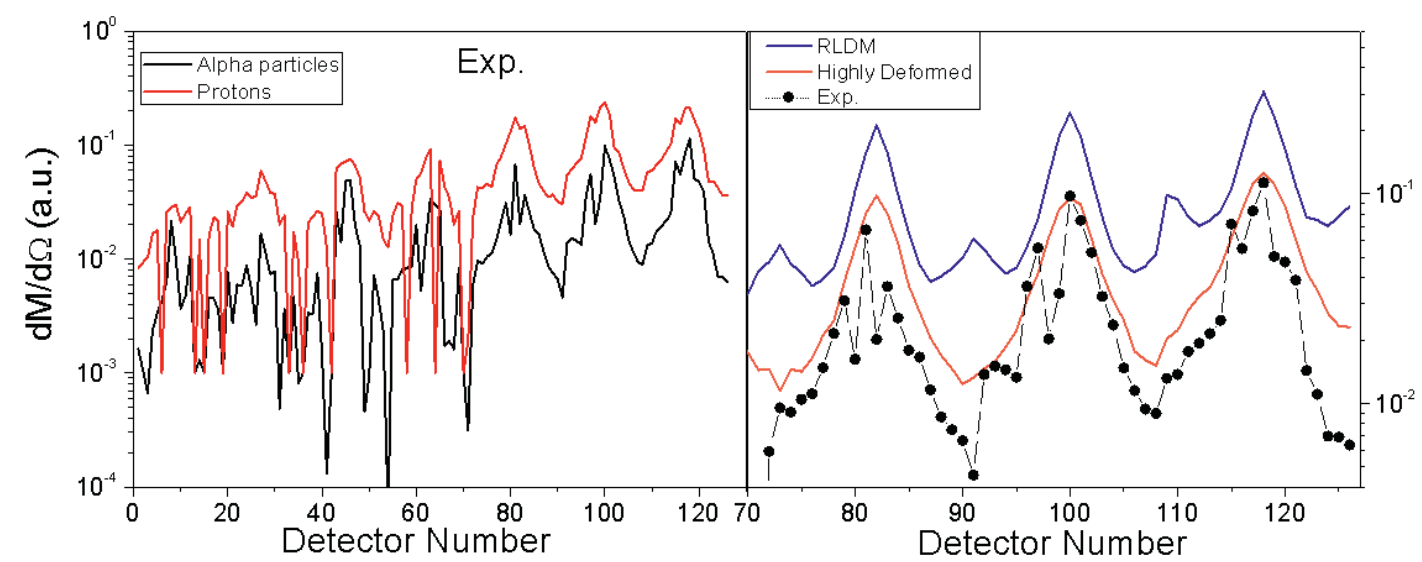

Fig. 4. Experimental angular multiplicity distributions for proton and alpha particles in coincidence with ERs versus the identification number of the BALL detectors (left side). Experimental alpha - ER coincidences compared to the simulation ones for rings $\mathrm{E}, \mathrm{F}$ and $\mathrm{G}$ of the BALL (right side).

the presence of a large deformation in this alpha-like system, that will be quantitatively determined after a complete analysis of the data. In the fig. 3 we show the ToF spectrum of the fragments detected by a PPAC sector collected with the Veto requirement (dark line) and in coincidence with the LCPs detected in the BALL (red line). ToF spectra were collected using the the Radio Frequency signal of the pulsed beam as a reference time. It should be noted that the LCP coincidence suppress the elastic and anelastic contributions.

In the fig. 4 we present the experimental $E R-L C P$ differential multiplicities for protons and alphaparticles, as in fig. 2. The proton multiplicity appears higher than that of the alpha-particles and the comparison with the simulations of fig. 2 supports the presence of a large deformation. In the right side of fig. 4, we present the $E R$ - alpha experimental angular correlation compared to the simulated 


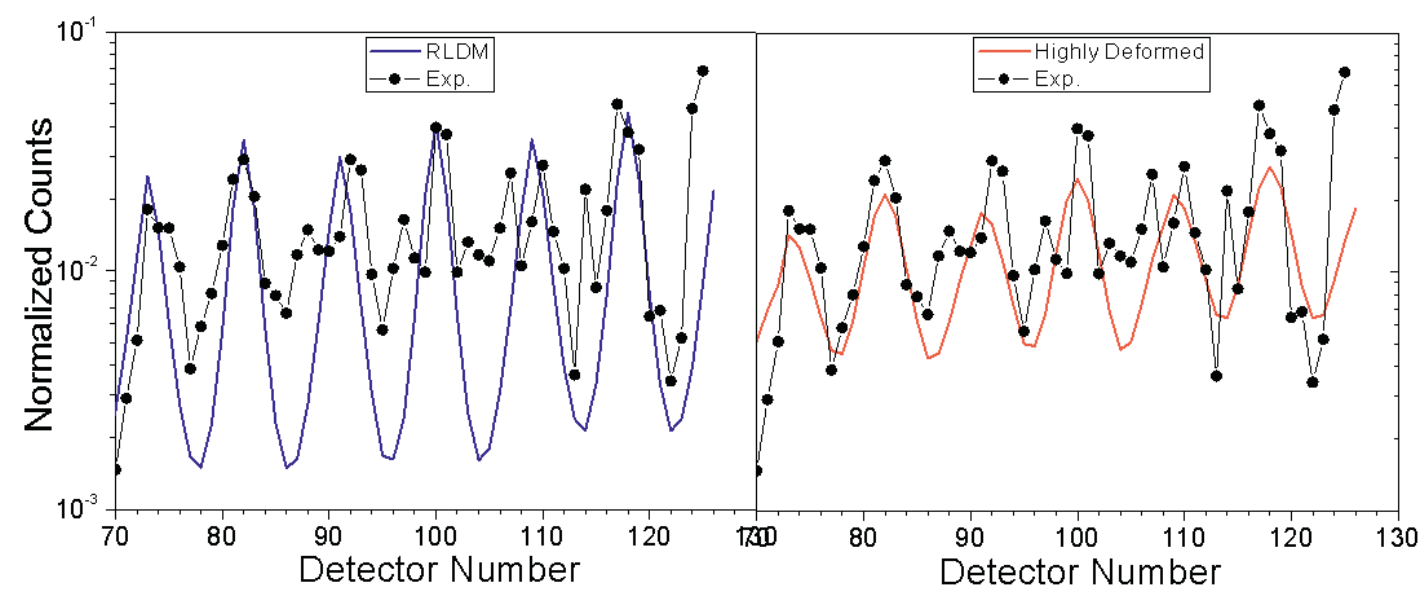

Fig. 5. Experimental angular multiplicity distribution of alpha particles in coincidence with the alpha particles observed at $\theta_{\mathrm{lab}}=42.7^{\circ}$ in the vertical plane above the beam, versus the identification number of the BALL detectors compared to RLDM (left side) and Highly Deformed (right side) simulations.

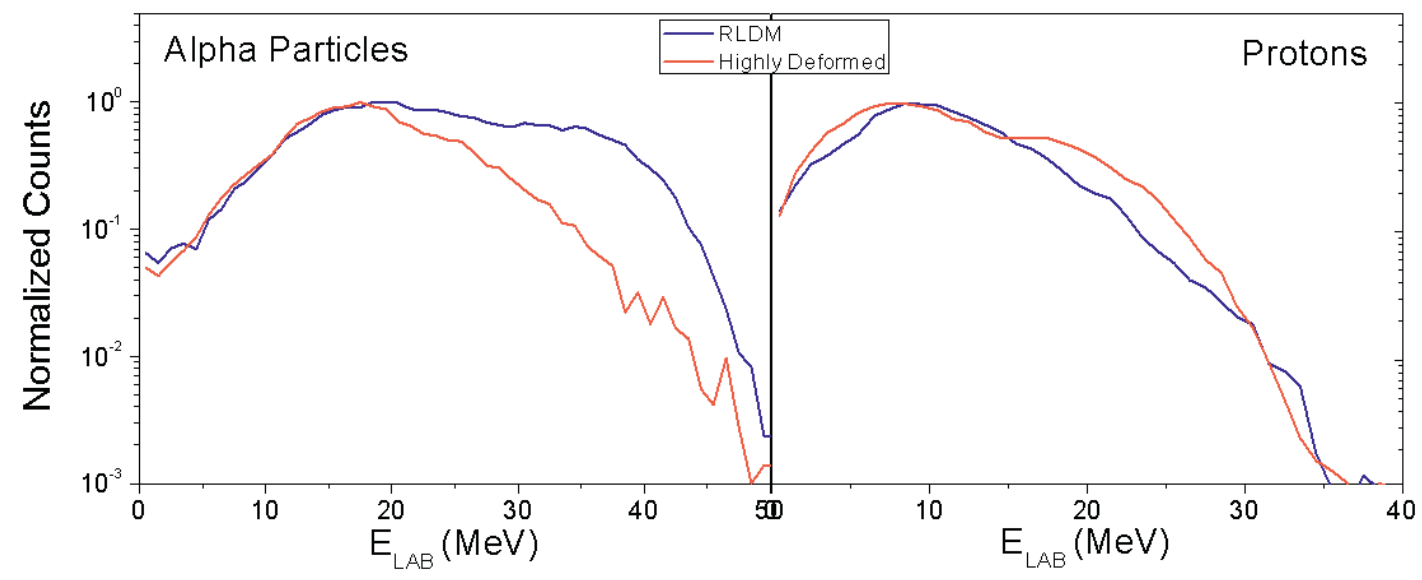

Fig. 6. Energy spectra of alpha particles and protons simulated at $\theta_{\mathrm{lab}}=42.7^{\circ}$ in coincidence with ERs.

RLDM and Highly Deformed ones for the E, F and G rings. We observe that the small peaks peculiar of the RLDM calculations are not present in the experimental data and in addition, the maximum to minimum ratio, in a fixed ring, is close to the value of the Highly Deformed simulation.

In addition, we measured the alpha particle events in coincidence with the alpha particles observed at $\theta_{\text {lab }}=42.7^{\circ}$ in the vertical plane above the beam. In the fig. 5 we present the alpha - alpha angular correlations versus the identification number of the BALL detectors compared to the SM simulations. We note a better agreement between the Highly Deformed simulation and the experimental data.

In conclusion, the preliminary analysis of ER-LCP and alpha-alpha angular correlations provide a good indication of the presence of a large deformation of the ${ }^{48} \mathrm{Cr}$ system. In order to get more information we will look at all the observables, which can be extracted from the measured data. In particular, energy spectra can be obtained and compared to the theoretical ones, as it was done in previous works. In fact, as it can be seen in the fig. 6 the two simulations of the alpha particle spectra, at $\theta_{\text {lab }}=42.7^{\circ}$, show a significant difference.

Moreover, we want to study the angular correlations for a selected decay channel. Considering the peculiarities of our apparatus a candidate for this study is the 3 alpha channel. SM simulations give an intensity of about $50 \mathrm{mb}$ for this channel with a low contamination from other channels. 
$\mathrm{CNR} * 11$

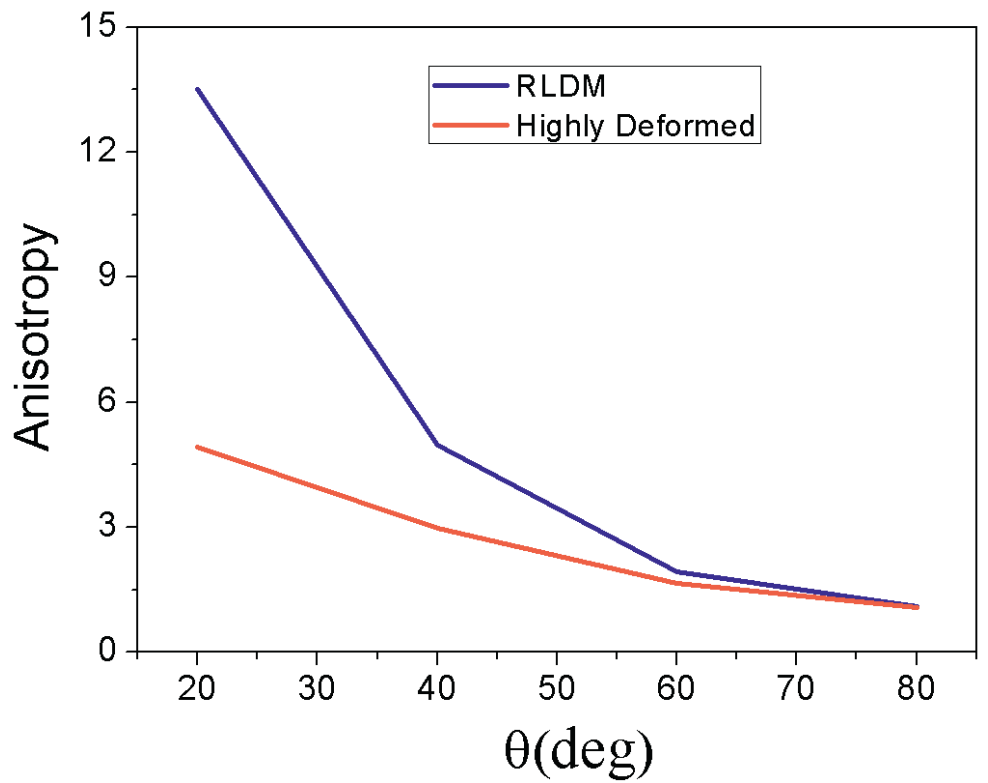

Fig. 7. Out of plane alpha particle correlation with respect to the plane defined by the detection of the two other alpha particles..

In fig.7 we present the comparison of the simulated correlations of an alpha particle emitted at the out-of-plane angle $\theta$, with respect to the plane defined by the detection of the two other alpha particles. The two prescriptions provide very different anysotropies which can be measured also with a low statistics.

\section{Conclusions}

We measured LCPs in coincidence with ERs for the composite system ${ }^{48} \mathrm{Cr}$ at $\mathrm{E}_{\mathrm{x}}=60 \mathrm{MeV}$ in order to explore on clustering effects related to the presence of the resonance with $\Gamma \approx 170 \mathrm{keV}, \mathrm{J}=36 \hbar$.

A preliminary analysis of the ER-LCP and LCP-LCP angular correlations, in the framework of the $\mathrm{SM}$, indicates the presence of a deformation much higher than the prediction of RLDM, which could be related to the presence of the resonance.

Statistics of the data will be improved taking advantage of the cylindrical symmetry of the $8 \pi \mathrm{LP}$ Ball and of the PPAC system. Moreover, the analysis of the WALL data will enlarge the angular coverage of the LCP correlations. The analysis of the energy spectra as well as of the angular correlations for selected LCP channels compared to the statistical model simulations will be very useful to provide additional constraints for the determination of the deformation.

\section{References}

1. H. Horiuchi et al., Nucl. Phys. A722, (2003), 80c

2. H. Horiuchi et al., Nucl. Phys. A731, (2004), 329

3. S. Thummerer et al., J. Phys. G27, (2001), 1405

4. S. J. Sanders et al., Phys. Rep. 311, (1999), 487

5. C. Bhattacharya et al., Phys. Rev. C65, (2001), 014611

6. Aparajita Dey et al., Phys. Rev. C74, (2006), 044605

7. M. Rousseau et al., Phys. Rev. C66, (2002), 034612

8. M. Norrby et al., Eur. Phys. J. A47, (2011), 96 doi 10.1140/epja/i2011-11096-1 
9. A.J. Sierk, Phys. Rev. C33, (1986), 2039 doi: 10.1103/PhysRevC.33.2039

10. R. W. Zurmuhle et al., Phys. Lett. B129, (1983), 384

11. A. H. Wuosmaa et al., Phys. Rev.C41, (1990), 2666

12. M.-D. Salsac et al., Nucl. Phys.A801, (2008), 1

13. E. Uegaki, Y. Abe, Phys. Lett. B231, (1989), 28

14. K. Pomorski and J. Dudek, Phys. Rev. C67, (2003), 044316

15. C.M. Jachcinski et al. Phys. Rev. C24, (1981), 2070

16. F.W. Prosser et al. Phys. Rev. C40, (1989), 2600

17. E. Fioretto et al. IEEE Trans. Nucl. Sci. 44, (1997), 1017

18. Lilita program was written by J. Gomez del Campo and R. G. Stockstad, Oak Ridge National Laboratory Report No. TM7295, 1981 (unpublished)

19. A. Gavron, Phys. Rev. C21, 230 (1980) doi: 10.1103/PhysRevC.21.230

20. J.R. Huizenga and G. Igo, Nucl. Phys. 29, 462 (1961)

21. F.G. Perey, Phys. Rev. 131, 745 (1963) doi: 10.1103/PhysRev.131.745

22. D. Willmore, P.E. Hudson, Nucl. Phys. 55, 673 (1964)

23. L.C. Vaz et al., Z. Phys. A318, 231 (1984)

24. A. Gilbert, A.G.W. Cameron, Can. J. Phys. 43, 1446 (1965)

25. W. E. Parker et al., Phys. Rev. C41, (1991), 774 